\title{
A PROPOS DE L'ACARE DE LA GALE NORVÉGIENNE
}

\author{
Par A. H. MANDOUL
}

Professeur de Parasitologie à la Faculté de médecine de Bordeaux

Divers auteurs se sont évertués à reconnaître à l'acare de la gale norvégienne des caractères spéciaux, sinoni spécifiques. Fürstenberg différencie cet acare de celui de la gale commune par la taille des adultes et des œufs et par la dimension des épines dorsales. Plus récemment, A. Buxton invoque les seuls caractères tirés des épines dorsales. Pour Mégnin, l'acare de la gale norvégienne est celui-là même qu'il a découvert sur les loups de la ménagerie du Muséum. Tous les auteurs sont loin d'ailleurs de partager cette manière de voir et nombreux sont ceux qui voient la dualité des parasites des deux formes de la gale humaine.

L'article de Ch. Joyeux paru ici-même et l'observation plus récente de W. Dubreuilh et Flye-Sainte-Marie apportent à l'appui de la théorie uniciste des arguments qui semblent décisifs. "Le Sarcopte (de la gale norvégienne), dit Ch. Joyeux, ne m'a paru présenter aucune différence morphologique avec Sarcoptes scabiei (L., 1748) var. hominis Mégnin. Cette constatation jointe aux cas de contagion observés par A. Pozzo et relatés ci-dessus, plaide en faveur de la théorie uniciste soutenue par Hébra et divers auteurs. " (T. I, p. 169).

L'observation de W. Dubreuilh et Flye-Sainte-Marie est non moins suggestive. Les acares, recueillis sur la malade entrée dans le service du professeur W. Dubreuilh à Bordeaux, m'ont fourni un matériel d'étude abondant. L'examen de ces parasites que j'ai pratiqué au Laboratoire avec le concours de M. Castebert, préparateur du service de Parasitologie et sur la demande de mon collègue, nous a donné des résultats suivants :

$1^{\circ}$ Máles : Longueur de $190 \mu$ à $195 \mu$; largeur $160 \mu$.

$2^{\circ}$ Femelles ovigères: Longueur de $350 \mu$ à $400 \mu$; largeur de $260 \mu$ à $300 \mu$. Les épines dorsales de la femelle sont bifurquées au sommet comme chez le sarcopte de la gale ordinaire.

$3^{\circ}$ Eufs : Longueur $150 \mu$; largeur $100 \mu$.

Aucun caractère ne permet de différencier ces sarcoptes de ceux de la gale commune, en particulier ceux tirés de la taille des adultes et des œufs ainsi que ceux invoqués par quelques auteurs, Fürsten-

Annales de Parasitologie, T. III, No 4. - Octobre 1925, p. 394-395. 
berg et plus récemment $\mathrm{A}$. Buxton. Les mensurations prises par Buxton concernant les épines dorsales présentent de telles variations qu'il ne paraît pas possible de déterminer leur longueur d'une façon précise en raison de leur obliquité.

Avec Ch. Joyeux nous concluons que les deux sarcoptes ne présentent aucune différence morphologique.

Il est curieux de constater que les sarcoptes de la gale norvégienne vivent dans les squames à la façon des acariens détriticoles, comme les tyroglyphes, par exemple, dans les croûtes du fromage.

Le cas de W. Dubreuilh est en outre intéressant par les nombreuses contagions familiales et hospitalières que la malade a répandues autour d'elle et qui ont toutes revêtu l'aspect habituel de la gale vulgaire. Je citerai parmi les victimes de l'entourage de la malade, son mari et sa fille avec lesquels elle vivait ; plusieurs de leurs voisines contaminées par la fille ; à l'hôpital les quelques malades de la salle qui ont contribué à la soigner ; les infirmières qui se sont occupées d'elle et enfin l'interne de service.

\section{RÉSUMÉ}

Ainsi done la cause paraît bien définitivement jugée. Gale norvégienne et gale commune reconnaissent pour agent pathogène un seul et même acarien : Sarcoptes scabiei (L., 1748) var. hominis Mégnin. La gale norvégienne tire son originalité, non de la spécificité de son parasite, mais de la forme de ses lésions.

\section{BIBLIOGRAPHIE}

Buxton (A.). - On the Sarcoptes of man. Parasitology, XII, 1921, p. 146-151.

Dubreullh (W.) et Flye-Sainte-Marie. - Bull. de la Soc. de Dermatologie, 10 janvier 1924 , p. $45-56$.

Joyedx (Ch.). - Sur un cas de gale norvégienne en Afrique Occidentale. Annales de Parasitologie, I, no 2, juin 1923, p. 167-169.

Laboratoire de Parasit logie de la Facullé de médecine de Bordeaux. 